\title{
Key Machining Characteristics in Ultrasonic Vibration Cutting of Single-crystal Silicon for Micro Grooves
}

\author{
Junyun Chen ${ }^{\mathrm{a}, \mathrm{c}, *}$, Tianye $\mathrm{Jin}^{\mathrm{b}}$, Xichun Luo ${ }^{\mathrm{c}}$
}

${ }^{a}$ School of Mechanical Engineering, Yanshan University, Qinhuangdao, +86-066004, China

${ }^{b}$ Center for Precision Engineering (CPE), Harbin Institute of Technology, Harbin, +86-150001, China

${ }^{\mathrm{c}}$ Department of Design, Manufacture and Engineering Management, Faculty of Engineering, University of Strathclyde, Glasgow G1 1XJ, UK

*Corresponding author, Email: sophiacjy@ysu.edu.cn, TEL: +86-13398658304, Postal address: No.438, Hebei Avenue, Qinhuangdao, Hebei Province, China

\begin{abstract}
Structured complex silicon components have the potential to develop breakthrough applications in solar cells, biomedical engineering, microfluidics, and MEMS. As silicon is a typical brittle material, ultrasonic vibration diamond cutting has been approved as a promising method to achieve better cutting performance compared to other conventional methods. However, few studies have been conducted on the cutting of structured silicon surfaces by applying high-frequency 1D ultrasonic vibration cutting (UVC), which is expected to possess higher material remove rate. Thus, a detailed understanding of the machining mechanism is yet to be developed. In this study, a series of tests that involve cutting grooves on the silicon surface were first performed by applying UVC and using a single crystal diamond tool. The machined surface and chips were subsequently measured and analysed to evaluate the critical undeformed chip thickness, surface finish, and chip formation. The critical undeformed chip thickness of silicon was found to reach $1030 \mathrm{~nm}$ under a certain vibration amplitude. An array of micro grooves was generated at the plastic region with a surface roughness $\mathrm{Ra}$ as low as $1.11 \mathrm{~nm}$. Moreover, the material removal and chip formation mechanisms were discussed with the assistance of developing a model used for predicting the length of the tool vibration mark. The results revealed that the micro topography of continuous chips exhibited discontinuous clusters of lines with diameters of dozens of nanometres which was only composed of polysilicon. The model was proved to be able to predict tool marks with extremely low error. Thus, the impact of tool marks on the surface finish can be reduced and even eliminated with help of the model.
\end{abstract}

Keywords: Ultrasonic vibration cutting, Single-crystal silicon, Micro groove, Chip, Tool vibration mark

This is a peer reviewed, accepted author manuscript of the following research article: Chen, J-Y., Jin, T-Y., \& Luo, X-C. (2019). Key machining characteristics in ultrasonic vibration cutting of single crystal silicon for micro grooves. Advances in Manufacturing, 7(3), 303-314. https://doi.org/10.1007/s40436-019-00263-4 


\section{Introduction}

Structured complex silicon components have the potential to develop breakthrough applications in solar cells, biomedical engineering, microfluidics, and MEMS for enhancing the functionality and performance of silicon components [1-4]. Traditionally, chemical-etching and focused ion beam are used to fabricate micro-nano structured silicon surfaces, but their low machining efficiency makes them unsuitable for complex structures with feature sizes in the range of hundreds of micrometres [5-7]. Some researchers have tried to machine structured surfaces using ultrashort pulsed lasers owing to the high material removal rate [8-10]. Nevertheless, the surface finish requirements for such silicon components are generally high and components produced using these techniques cannot often meet these requirements. Additionally, the micro grinding method that is commonly used is not only time-consuming but also has limited ability to generate complicated surfaces [11-15]. Currently, ultra-precision diamond cutting technology is regard as a promising method of generating complex structures on the surface of brittle materials with better form accuracy and surface finish compared to other traditional methods. For instance, Mukaida et al. successfully fabricated concave microlens arrays with a form error of $300 \mathrm{~nm}$ PV and surface roughness of $6 \mathrm{~nm}$ Sa on a single-crystal silicon wafer by slow tool servo diamond turning [16]. However, a crack-free silicon surface can be generated in ductile region only by adopting an extremely small undeformed chip thickness because of its intrinsic low fracture toughness [17]. In addition, the tool wear in the diamond cutting of silicon is too serious to meet the requirements of structured complex components for industrial applications [18-19].

Ultrasonic vibration cutting (UVC) is regard as a better method of achieving longer tool life, lower cutting force, and better surface quality as compared with conventional cutting (CC) method [20-22]. For silicon materials, the critical undeformed chip thickness obtained by UVC was proved to be much larger than that resulting from CC [22-23]. UVC is generally classified into 1D and 2D/3D system according to the number of vibration directions [22,24]. The former was developed in the late 1950s for cutting metals. Later on, it was successfully applied in the ductile cutting of brittle materials [25]. 2D/3D UVC can generate lower forces and thinner chips, which is generally thought to be more beneficial to the ultraprecision cutting of brittle materials [22,24], but its vibration frequency is limited by the more complex structure and control system compared with that of 1D UVC [20-27]. Hence, the cutting speed and material removal rate of 1D UVC is higher than that of 2D/3D UVC, and thus, is more suitable for industrial application. Nowadays, 1D UVC is used in the industrial production of hardened steel dies for optical components, and it might be feasible for application in the manufacture of structured silicon components in the future.

However, few researchers have focused on ultraprecision cutting or structured surface machining of single-crystal silicon by the application of 1D UVC. Therefore, a detailed understanding of the machining performance and mechanism has not yet been developed. In this study, a commercial 1D UVC system with the frequency of $103 \mathrm{kHz}$ was adopted to study the ultra-precision diamond cutting of a structured silicon surface. The critical undeformed chip 
thickness and the influence of process parameters were first analysed through micro groove experiments. Subsequently, the material removal and chip formation mechanism were discussed. In order to reduce and eliminate the impact of tool vibration marks induced by 1D UVC on the surface finish, a model for predicting the length of the tool vibration mark was established to provide information that could be used to improve the surface quality of micro grooves.

\section{Experimental setup and procedures}

\subsection{Experimental setup}

A set of experiments involving the ultrasonic vibration cutting of silicon were conducted on a four-axis ultra-precision machine tool (Moore nanotech 450UPL) shown in Fig.1. The UVC system (UTS 2, Son-X, Germany) mounted on the B axis has a vibration frequency of $103 \mathrm{kHz}$. During the cutting of grooves, the system drove the cutting tool vibration mainly along the $\mathrm{X}$ direction, in addition to a movement of approximately $100 \mathrm{~nm}$ along the z-direction within one vibration cycle. A natural single crystalline diamond tool was fixed in the holder of the UVC system, which has a nose radius of $1.0 \mathrm{~mm}$, a rake angle of $0^{\circ}$, and a clearance angle of $7^{\circ}$. In order to improve the accuracy of tool setting, a digital microscope of magnification 400x was mounted above the cutting tool. The workpiece was a (100) single crystal silicon wafer of $\Phi 20 \mathrm{~mm} \times 5 \mathrm{~mm}$. It was glued on the work spindle after polishing.

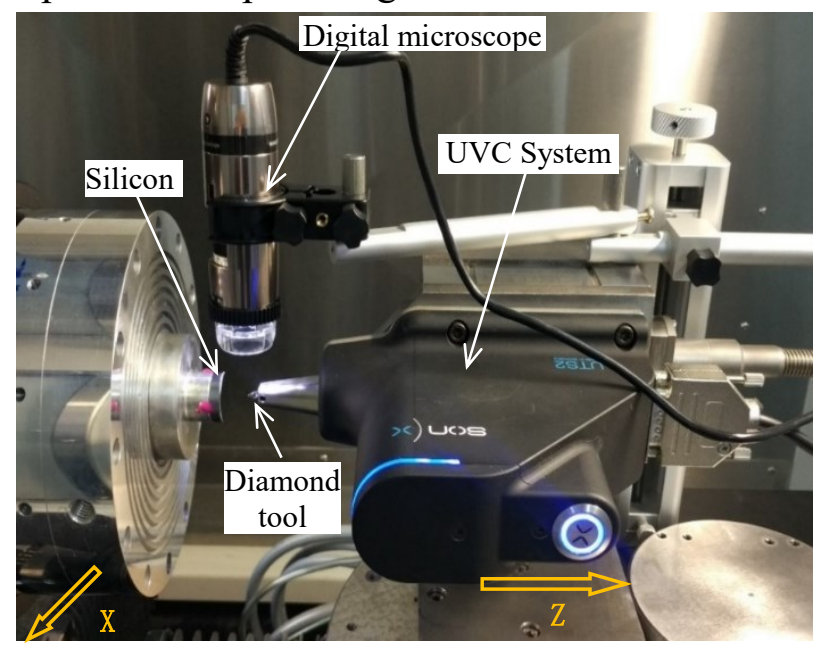

Fig.1. Experimental setup of cutting grooves with UVC system.

\subsection{Experimental procedures}

In order to study the critical undeformed chip thickness in the cutting of silicon with and without vibration assistance, micro sloping grooves were cut in and then cut out along the $\mathrm{x}$ direction with varying depth of cut. The maximum feed along the z-direction was $5 \mu \mathrm{m}$ and the cutting speed along the $x$-direction was set as $200 \mathrm{~mm} / \mathrm{min}$. Considering that vibration amplitude is a main parameter that affects the critical undeformed chip thickness, it was set as $0 \mathrm{~nm}, 590 \mathrm{~nm}$, $870 \mathrm{~nm}, 1026 \mathrm{~nm}$, and $1700 \mathrm{~nm}$, respectively, based on the available range of the system. The 
machined surface was first cleaned in alcohol with ultrasonic assistance. After cleaning, the morphology of the micro grooves was first using a Scanning Electron Microscope (SEM, Phenom Pro). Later, an Atom Force Microscope (AFM, Dimension ${ }^{\circledR}$ Icon $^{\mathrm{TM}}$ ) was used to measure the surface roughness and surface profile of the micro grooves.

The cutting conditions for the micro groove array are shown in Table 1. After cutting and cleaning, the machined surface was scanned by SEM to capture the morphology of the grooves. In addition, the surface roughness and profile were measured by AFM for each groove. The chips generated during groove cutting were collected and then scanned by SEM. For the sake of investigating possible material structural changes of the chip, laser micro-Raman spectroscopy (Renishaw, inVia) was performed on the chip surface with a laser power of $5 \mathrm{~mW}$.

Table 1 Cutting conditions for micro grooves

\begin{tabular}{cccc}
\hline Term & $\begin{array}{c}\text { Cutting speed } \\
v_{c}(\mathrm{~mm} / \mathrm{min})\end{array}$ & $\begin{array}{c}\text { Depth of cut } \\
d_{c}(\mathrm{~nm})\end{array}$ & $\begin{array}{c}\text { Vibration amplitude } \\
\text { No. }\end{array}$ \\
\hline 1 & 200 & 100 & 1000 \\
2 & 200 & 300 & 1000 \\
3 & 200 & 500 & 1000 \\
4 & 200 & 700 & 1000 \\
5 & 50 & 300 & 1000 \\
6 & 500 & 300 & 1000 \\
7 & 800 & 300 & 1000 \\
8 & 200 & 300 & 1350 \\
9 & 200 & 300 & 750 \\
10 & 200 & 300 & 480 \\
\hline
\end{tabular}

\section{Experimental results and discussion}

\subsection{Characteristics of sloping grooves}

The micro groove that was machined without vibration assistance is shown in Fig.2 (a) and (b). The micro topography showed that a great number of cracks and pits quickly appeared on the bottom of the groove with increasing depth of cut, where almost all the material was removed in the form of brittle fracture. On the other hand, with vibration assistance, the area of the smooth surface on each groove formed by plastic deformation was much larger than conventional cutting shown in Fig.2 (c)-(j). In addition, the density of cracks and pits on the surface obtained with UVC is much lower than in the $\mathrm{CC}$ within the region of the ductile-brittle transition. From the groove surface shown in Fig. 2, it can be seen that the width of the groove at the point of generating the earliest crack or pit gradually increases with the increase in vibration amplitude, which indicates that a larger vibration amplitude will result in a larger critical depth of cut $\left(d_{c}\right)$ and undeformed chip thickness $\left(t_{c}\right)$ for the ductile-brittle transition. 

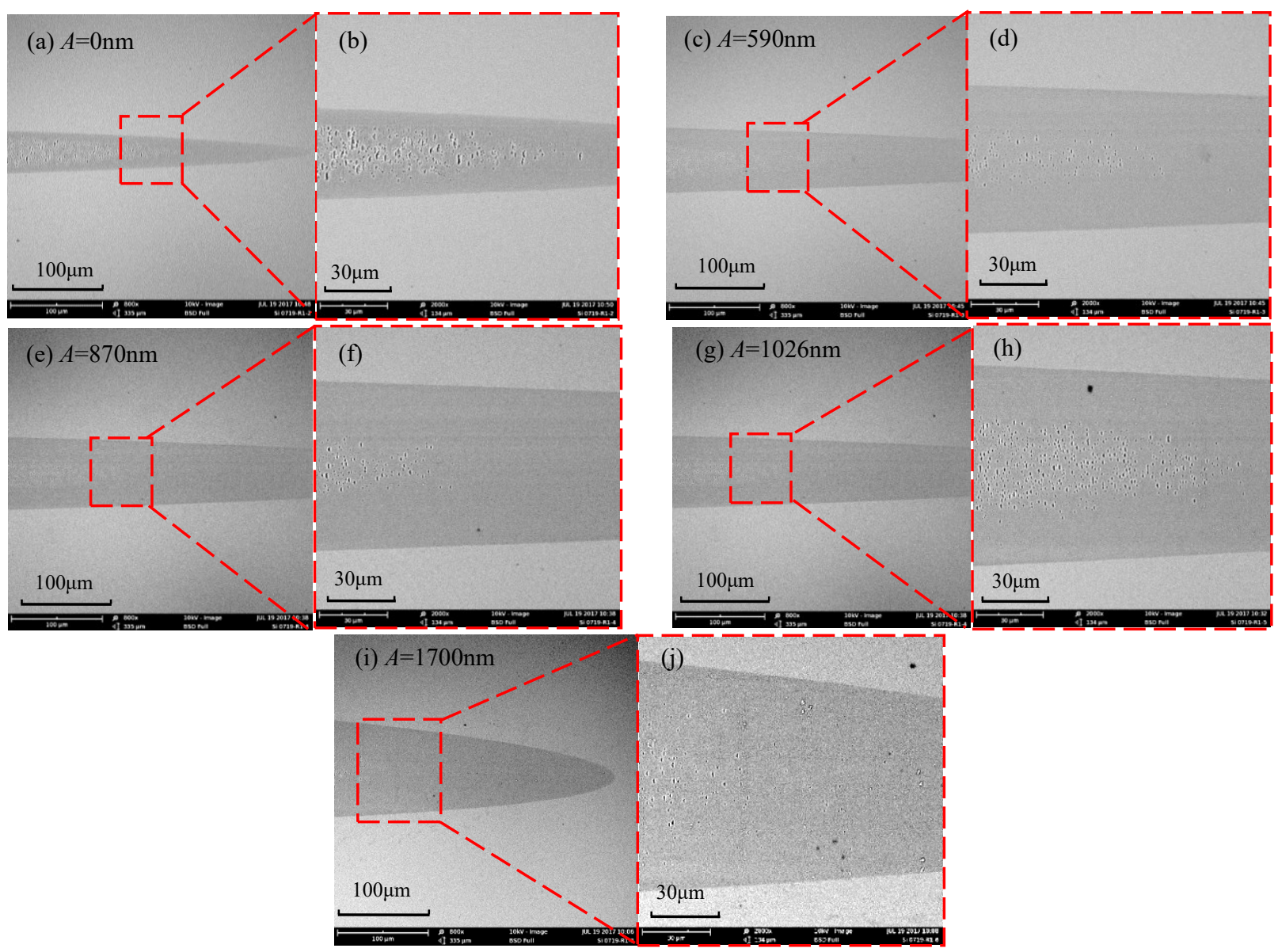

Fig.2. Morphology of sloping grooves captured by SEM with different vibration amplitude

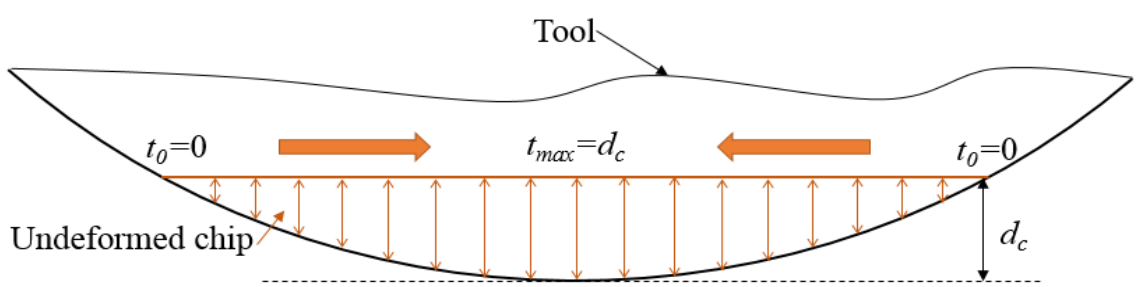

Fig.3. The relationship between depth of cut and undeformed chip thickness during cutting groove

As shown in Fig.3, the thickness of the undeformed chip $(t)$ increases from zero at the edge of the groove to a maximum value $d_{c}$ at the center. Thus, the critical undeformed chip thickness $t_{c}$ can be calculated according to the cross sectional profile of the groove at the position of ductilebrittle transition. Subsequently, the micro grooves in Fig. 2 were observed in more detail under AFM to find the position of ductile-brittle transition, and the topography of the position was scanned by AFM as shown in Fig.4 (left) to achieve the cross sectional profile in Fig.4 (right). However, for the vibration amplitude of $1700 \mathrm{~nm}$, the groove surface near the transition point is too deep to measure directly by AFM. Therefore, the left half part of the groove was scanned after tilting by an angle of $45^{\circ}$, and then the data points on the cross sectional profile were extracted, fitted, and corrected using MATLAB software to generate a profile after correction as shown in Fig.4 (e). The values of critical undeformed chip thickness can only reach $120 \mathrm{~nm}$ without 
vibration assistance. Nevertheless, under the same cutting parameters other than the vibration amplitude, the critical undeformed chip thickness grows from $400 \mathrm{~nm}$ to $1030 \mathrm{~nm}$ when the vibration amplitude increases from $590 \mathrm{~nm}$ to $1700 \mathrm{~nm}$. The maximum value of critical undeformed chip thickness is about nine times that achieved without vibration assistance. Obviously, the higher critical undeformed chip thickness obtained by applying 1D UVC, as compared to other vibration assisted machining systems [21-26], is significant to raise the machining efficiency of micro structured silicon components in the ductile region.

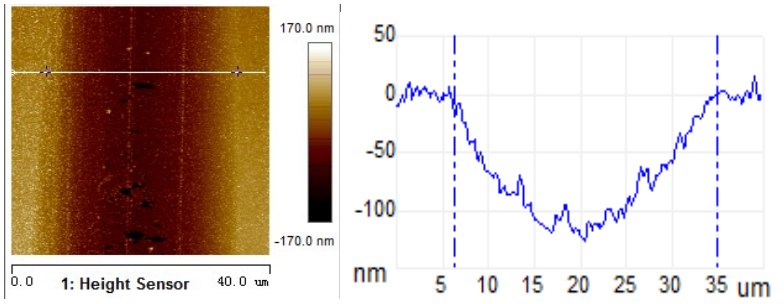

(a) $A=0 \mathrm{~nm}, t_{c}=120 \mathrm{~nm}$

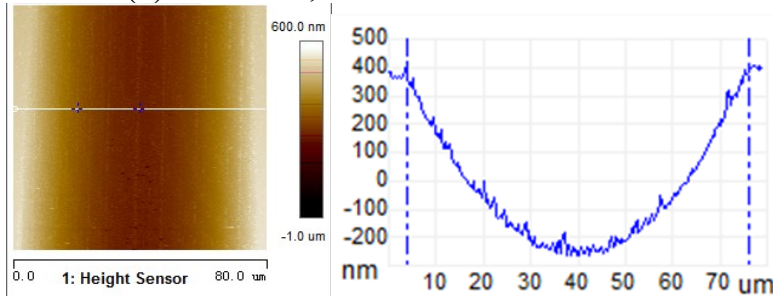

(c) $A=870 \mathrm{~nm}, t_{c}=630 \mathrm{~nm}$

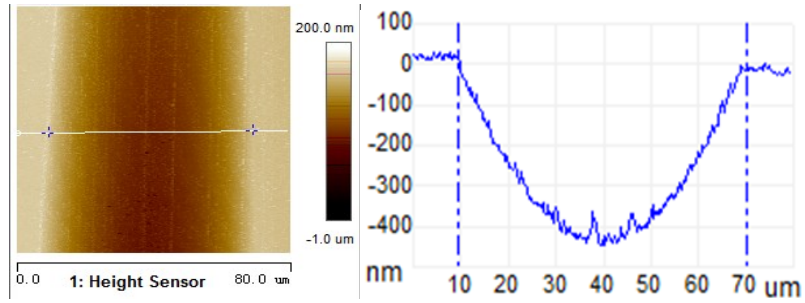

(b) $A=590 \mathrm{~nm}, t_{c}=400 \mathrm{~nm}$

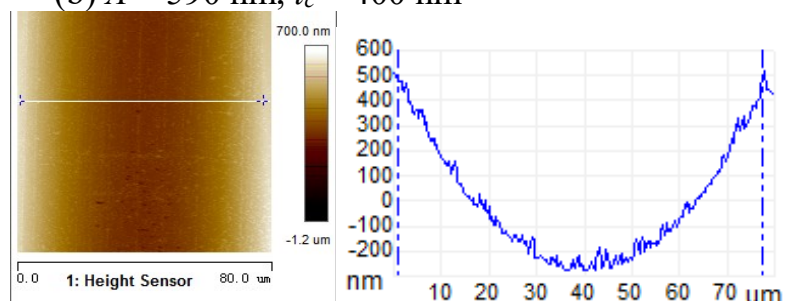

(d) $A=1026 \mathrm{~nm}, t_{c}=750 \mathrm{~nm}$

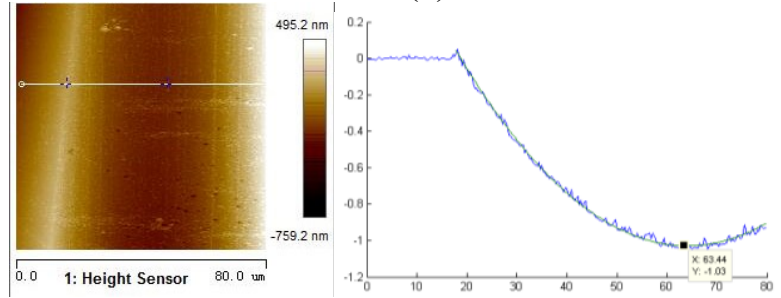

(e) $A=1700 \mathrm{~nm}, t_{c}=1030 \mathrm{~nm}$

Fig.4. Morphology of sloping groove measured by AFM (left) and extracted cross sectional profile at the position of ductile-brittle transition (right).

\subsection{Characteristics of micro groove array}

The tests involving micro groove arrays were conducted under the conditions summarized in Table 1, which was designed according to the results of critical undeformed chip thickness. As shown in Fig.5, micro grooves with complete profile were formed without any obvious cracks or pits on silicon surface, although the maximum depth of cut was set as deep as $700 \mathrm{~nm}$.
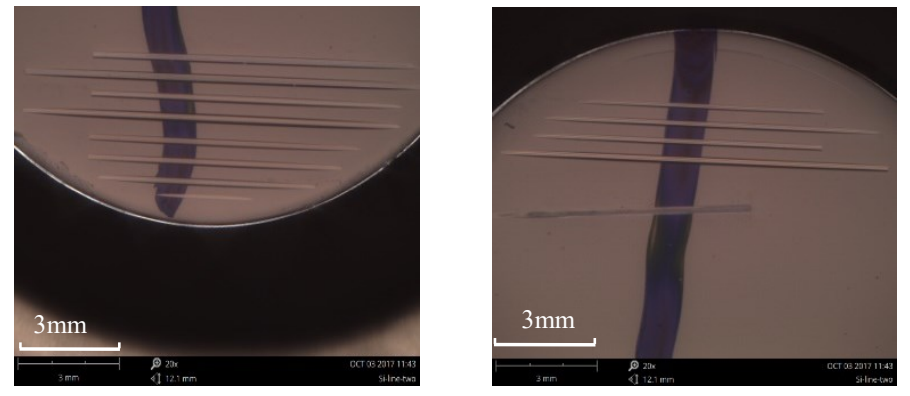
Fig.5. Micro grooves array on silicon surface captured by SEM.

Micro topography of micro grooves under varying depth of cut was further observed by SEM as shown in Fig.6. It is clear that smooth surface within all the machined surface was generated. There is no feature of brittle fracture along the edges of the grooves, in which it is easy to produce a crack under the influence of the chip. It is noted that a few of the nano-lines extending along the direction of cutting speed can be found on the groove surface. Moreover, the number of nano-lines increases when the depth of cut increases. Researchers found that the chemical reaction between carbon within the diamond and the silicon was unavoidable to generate hard particles of silicon carbide in the cutting of silicon using a diamond tool [18,19,28]. Therefore, the hard particles of silicon carbide were also formed in cutting micro grooves by the diamond tool. The particles flowed into the tool-chip interface and then cut the groove surface in a manner similar to abrasive grains in lapping. Finally, nano-lines were formed at the ductile region on the machined surface.

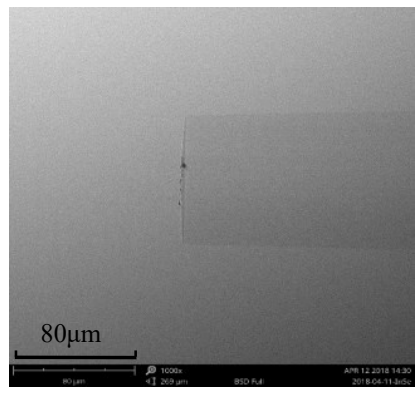

(a) $d_{c}=100 \mathrm{~nm}$

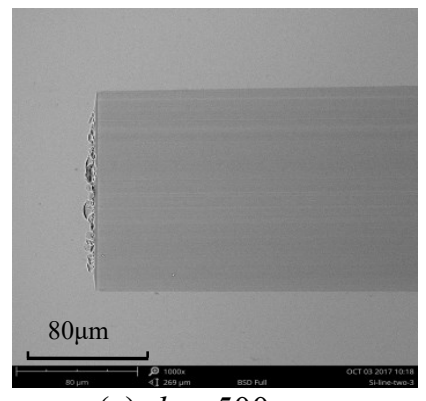

(c) $d_{c}=500 \mathrm{~nm}$

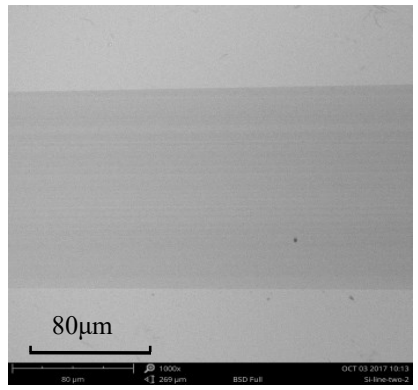

(b) $d_{c}=300 \mathrm{~nm}$

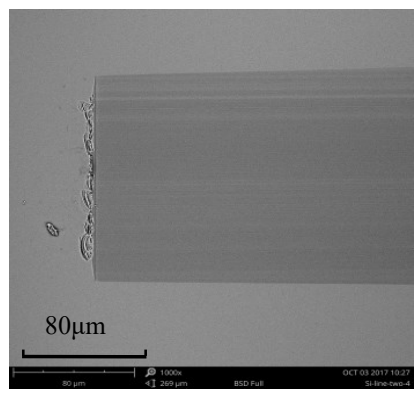

(d) $d_{c}=700 \mathrm{~nm}$

Fig.6. Topography of micro groove array captured by SEM under varying depth of cut.

On the basis of 2D and 3D topography imaged by AFM, the surface roughness was calculated for each groove after flattening, as shown in Fig.7. The average surface roughness is approximately $2 \mathrm{~nm}$, which is slightly affected by depth of cut. Furthermore, surface roughness of grooves raises from $4.1 \mathrm{~nm}$ to $6.17 \mathrm{~nm}$ as increasing cutting speed, but it is as low as $1.11 \mathrm{~nm}$ at the speed of 200 $\mathrm{mm} / \mathrm{min}$. It can be explained that a cutting speed lower than $200 \mathrm{~mm} / \mathrm{min}$ led to the adverse plastic deformation of the silicon material as well as bad removal of chip. On the other hand, a high cutting speed might cause tool vibration marks to appear on the machined surface, which will be analyzed in more detail in the next part of this paper. From the surface roughness values in Fig.7 (c), it can be seen that the surface roughness goes up when the vibration amplitude is higher than $1000 \mathrm{~nm}$ which is caused by the longer contact time between tool and sample material during a single 
vibration cycle when compared with lower vibration amplitudes. In this case, more silicon carbide particles were retained in the tool-chip interface due to worse lubrication between the tool and the chip, which produced more nano-lines distributed on the groove surface and worsened the surface finish than in the case of a lower vibration amplitude. From the surface roughness measurements, it can be concluded that a crack free surface can be obtained only if the depth of cut is lower than the critical undeformed chip thickness and the effect of depth of cut on the surface finish is negligible. Additionally, a higher cutting speed or vibration amplitude is not beneficial to improving the surface quality.

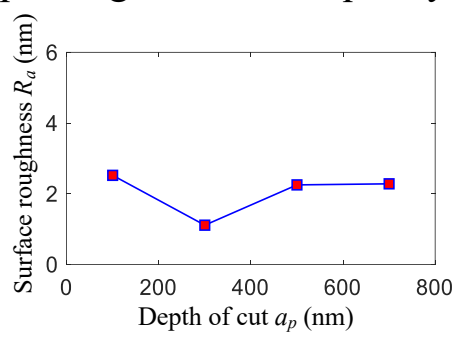

(a) Depth of cut

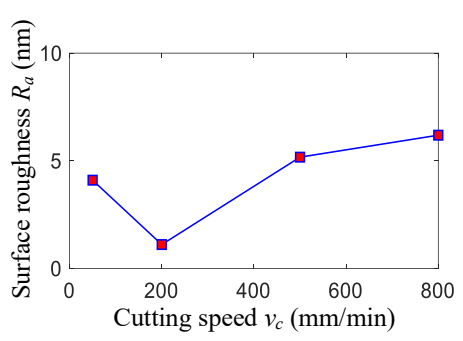

(b) Cutting speed

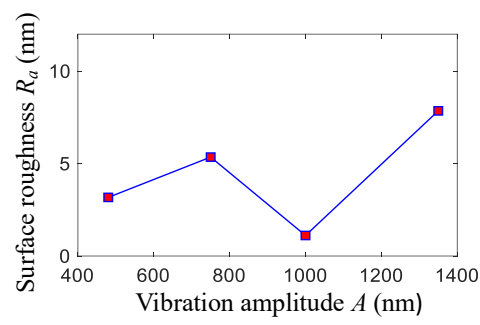

(c) Vibration amplitude

Fig.7. Surface roughness of micro grooves with the variation of process parameters.

\section{Analysis of material removal process and mechanism}

\subsection{Formation of chip}

During the cutting of micro grooves on a silicon surface, the generated chips seemed continuous, complete, and unbroken. When the chips were subject to further observation under SEM, its micro topography indicated that it is not completely continuous unlike the macro image of the chips shown in Fig. 8 . The breakage in the chip might be caused by the interrupted cutting process in vibration assisted machining.

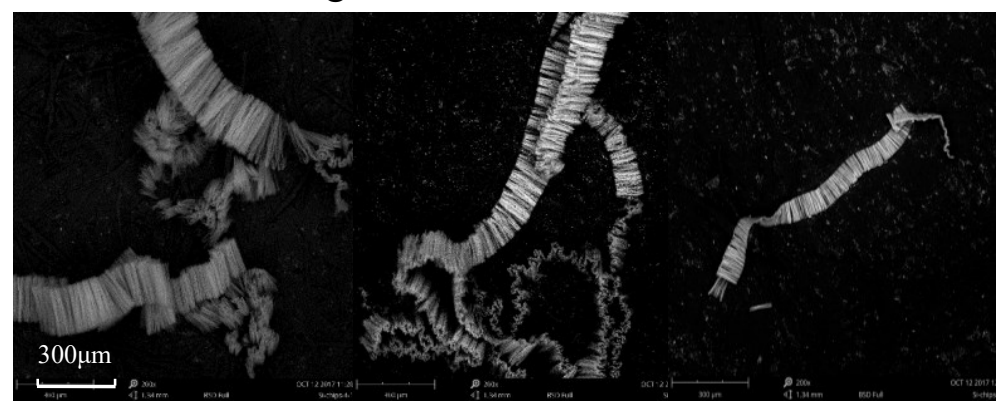

Fig.8. Morphology of chips imaged by SEM with low magnification.

In the case of the 5000x magnification under SEM, the chips appeared as having clusters of lines with diameter of the order of dozens of nanometres as shown in Fig.9 (a), which indicated that one linear chip was generated for each vibration cycle. With the movement of the cutting tool, a large number of linear chips were piled up to the clusters of lines, which made them appear continuous. As shown in Fig.9 (b), the chips collected from a groove machined with a depth of cut of $300 \mathrm{~nm}$ and vibration amplitude of $480 \mathrm{~nm}$ was found to be different from the chips from other grooves, and it appeared like clusters of thin slices. In this instance, the critical undeformed chip 
thickness of $380 \mathrm{~nm}$ was determined based on the relationship between the critical undeformed chip thickness and vibration amplitude. Therefore, it can be explained that the chip for each vibration cycle cannot maintain linear integrity, but was piled up into thin slices when the maximum thickness of the undeformed chip became close to the critical value for the ductile-brittle transition under certain process parameters. However, the formation of chips characterized by clusters of thin slices was not harmful to the surface finish of the groove.

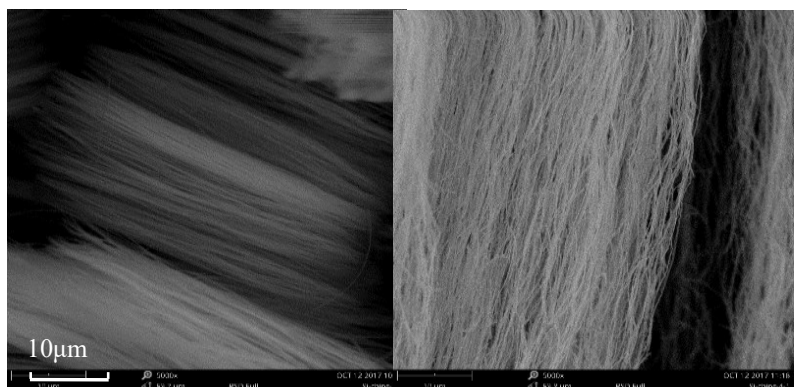

(a) Chips featured by clusters of lines

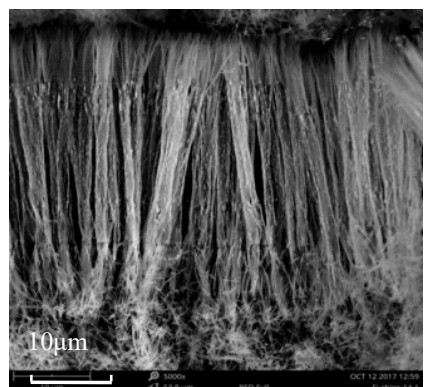

(b) Chips featured by clusters of thin slices

Fig.9. Morphology of chips imaged by SEM with high magnification.

Raman spectroscopy can be used to distinguish the different forms of silicon. As shown in Fig.10, all the Raman spectra obtained under the variation of process parameters presents a sharp intense band near the point at $500 \mathrm{~cm}^{-1}$ which can be assigned to polysilicon, as the sharp Raman line was located between that corresponding to amorphous silicon ( $\alpha$-Si) at $470 \mathrm{~cm}^{-1}$ and single crystal silicon (c-Si) at $520.7 \mathrm{~cm}^{-1}[16,29,30]$. The Raman shift to a slightly higher wave number indicates a compressive residual stress on the chips obtained with the higher cutting speed of 800 $\mathrm{mm} / \mathrm{min}$ and lower cutting speed of $50 \mathrm{~mm} / \mathrm{min}$ [16,29], as shown in Fig.10 (b), that might arise from increasing the applied pressure when chips cannot flow out of the tool-chip interface smoothly. This may help explain why the higher or lower cutting speed slightly increases the surface roughness shown in Fig.7 (b). Mukaida, et al. found that both amorphous and polycrystalline chips were generated in the ductile cutting of silicon without vibration assistance [16], but only poly-crystalline chips were detected in vibration assisted machining. Past researchers found that for unloading rates faster than $1 \mathrm{~mm} / \mathrm{min}$, only amorphous silicon was formed under the indentation during indentation experiments [30]. Cutting force with vibration assistance was deduced to be lower than that in CC as presented by Mukaida [16,21], and the strain rate during chip formation was too low to generate amorphous silicon.

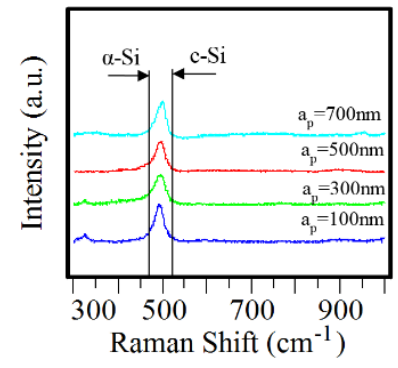

(a) Depth of cut

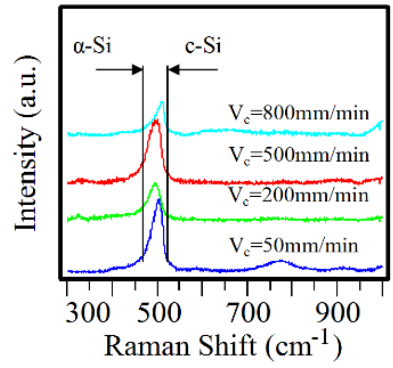

(b) Cutting speed

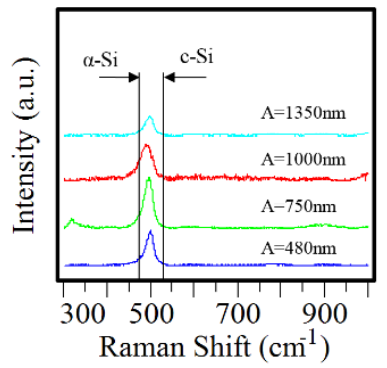

(c) Vibration amplitude

Fig.10. Raman spectra of chips collected under different process parameters. 


\subsection{A model of material removal length}

As the main vibration direction of the 1D UVC system was the same as cutting speed, the motion of tool can be simplified as vibration moving between the highest vibration position (HVP) and lowest vibration position (LVP) as shown in Fig.11. During one vibration cycle, point (1) was assumed to be the starting position as shown in Figs.11 and 12. At this moment, the sample moved downwards while the motion of tool was towards the sample. Then, the relative distance between the tool and sample became smaller and smaller until it became zero at point (2), from which material removal occurred. After point (3), the speed of tool $\left(v_{t}\right)$ gradually increased from zero, but it is smaller than the speed of the sample $\left(v_{c}\right)$ which also resulted in material removal in the sample. At point (4), the relative speed between the tool and sample equaled zero which means that the tool would go away from the sample with increasing speed. Then, the tool moved away from the sample and its downward motion turned into upward motion at point (5). Finally, the tool moved upwards with increasing speed until point (1) to finish a complete cycle and commence the next one.

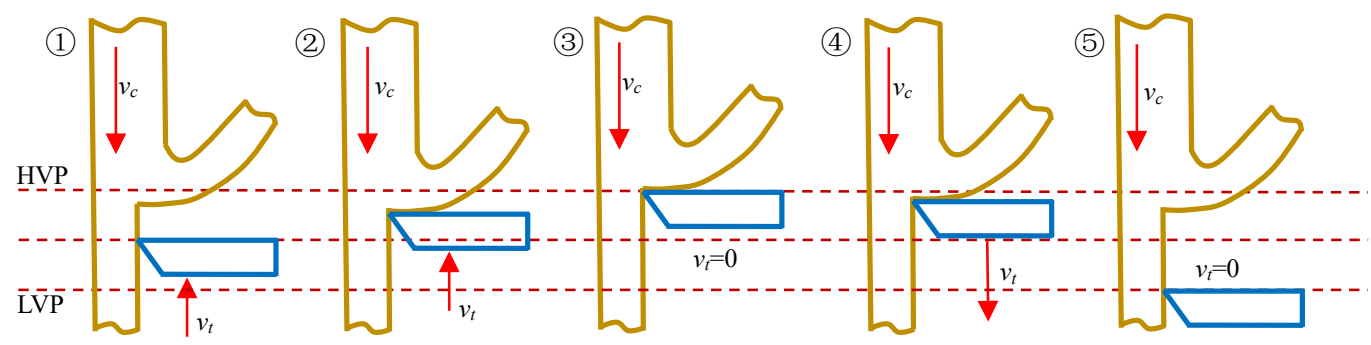

Fig.11. Tool and sample motion in one vibration cycle.

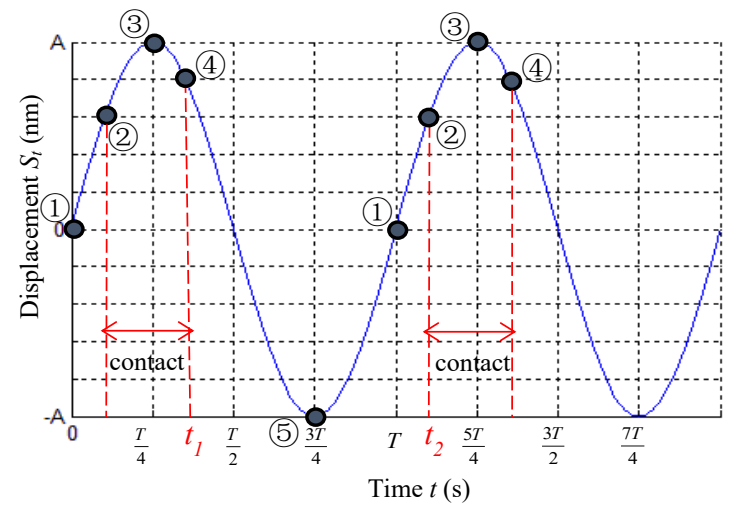

Fig. 12. Vibrating curve of cutting tool.

The motion of tool was simplified as a sine function shown in Fig.12, as follows

$$
S_{t}=A \sin (2 \pi f t)
$$

where $S_{t}$ is the displacement of the tool, $A$ is vibration amplitude and $f$ is vibration frequency. Differentiating, the speed of tool $v_{t}$ can be expressed as

$$
v_{t}=2 \pi f A \cos (2 \pi f t)
$$


within the contact time between the tool and sample, the time range from point (3) to (4) was first considered in order to calculate the material remove length conveniently, since the speed of tool at point (3) equals zero. As mentioned above, the relative speed between the tool and simple decreased to zero at point (4), resulting in $v_{c}=v_{t}$. Then, the time $t_{1}$ when tool moved away from the sample can be obtained as

$$
t_{1}=\frac{T}{2}-\cos ^{-1}\left(\frac{v_{c}}{2 \pi f A}\right)
$$

within the time range from $\frac{T}{4}$ to $t_{1}$, the movement distance of sample was expressed as

$$
S_{c 1}=v_{c}\left[\frac{T}{4}-\cos ^{-1}\left(\frac{v_{c}}{2 \pi f A}\right)\right]
$$

while the movement distance of the tool was

$$
S_{t 1}=A-A \sin \left(2 \pi f t_{1}\right)
$$

The material removal length $S_{1}$ from point (3) to (4) can be obtained as

$$
S_{1}=S_{c 1}-S_{t 1}=v_{c}\left[\frac{\pi}{4}-\cos ^{-1}\left(\frac{v_{c}}{2 \pi f A}\right)\right]-\left\{A-A \sin \left[2 \pi f\left(\frac{T}{2}-\cos ^{-1}\left(\frac{v_{c}}{2 \pi f A}\right)\right)\right]\right\}
$$

Subsequently, the tool was separated from the sample because of $v_{c}<v_{t}$ with the downward movement after point (4), and then it moved towards the sample to the end point of this cycle that was also the starting point of next cycle (point (1)) via point (5). At the moment $t_{2}$ shown in Fig.11, the tool moving upwards contacted the sample with a downward movement. At this point, the displacement of the tool in Fig. 12 can be expressed as

$$
S_{t-c o n}=A \sin \left(2 \pi f t_{2}\right)
$$

while the movement distance of sample from the point (3) of the last cycle to point (2) of this cycle was as follows

$$
S_{c-c o n}=v_{c}\left(t_{2}-\frac{T}{4}\right)
$$

Because the tool makes contact with the sample, we can say that

$$
S_{t-c o n}+S_{c-c o n}=A
$$

Thus, $t_{2}$ can be calculated from the following equation

$$
A \sin \left(2 \pi f t_{2}\right)+v_{c}\left(t_{2}-\frac{T}{4}\right)=A
$$

within the time range from $t_{2}$ to $\frac{5 T}{4}$, material removal occurred again. During this period, the movement distance of sample was given as 


$$
S_{c 2}=v_{c}\left(\frac{5 T}{4}-t_{2}\right)
$$

while distance of tool movement was

$$
S_{t 2}=A-A \sin \left(2 \pi f t_{2}\right)
$$

and the material removal length in this period was expressed as

$$
S_{2}=S_{c 2}+S_{t 2}
$$

As shown in Fig.12, material was removed from the sample within the time range from point (2) to point (4) for one vibration cycle. Therefore, the material removal length can be calculated as

$$
S=S_{1}+S_{2}
$$

\subsection{Verification of the model}

Previous research indicated that tool vibration trace was inevitably imprinted on machined surface to form tool vibration mark if the main direction of vibration was along that of the cutting speed, especially for 1D UVC [21]. However, the tool vibration mark was rarely studied despite its prominent effect on the surface roughness. In this study, material removal corresponding to each vibration cycle was considered to leave one mark on the machined surface. Hence, the material removal length in one cycle theoretically coincided with the length of the tool vibration mark, which can be predicted by the model presented in this paper.

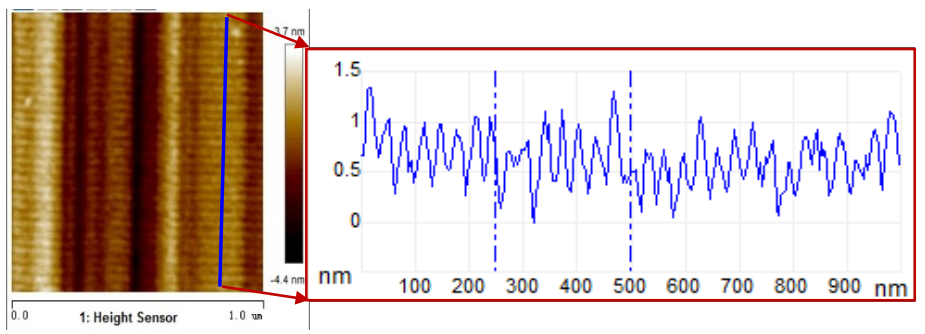

(a) $v_{c}=200 \mathrm{~mm} / \mathrm{min}, a_{p}=100 \mathrm{~nm}, A=1000 \mathrm{~nm}$

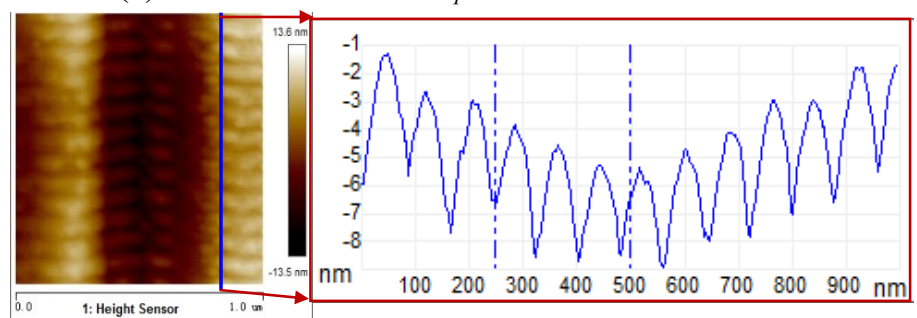

(b) $v_{c}=500 \mathrm{~mm} / \mathrm{min}, a_{p}=300 \mathrm{~nm}, A=1000 \mathrm{~nm}$ 


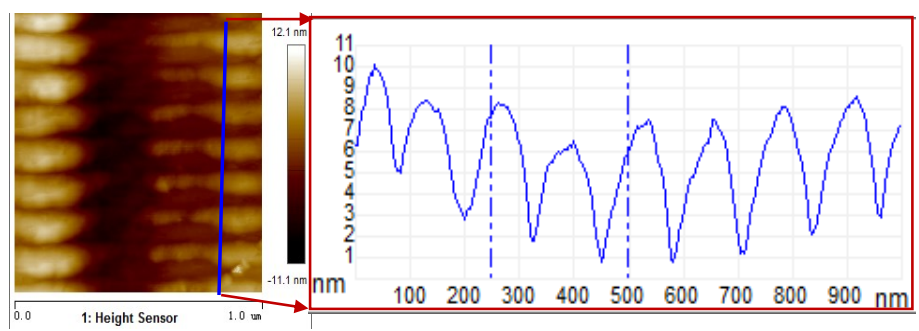

(c) $v_{c}=800 \mathrm{~mm} / \mathrm{min}, a_{p}=300 \mathrm{~nm}, A=1000 \mathrm{~nm}$

Fig.13. Tool marks on the groove surfaces.

By observing the surface topography imaged by AFM on the groove surfaces machined under the conditions listed in Table 1, almost all the surfaces exhibited tool vibration marks. Then, the cross sectional profile was extracted along the direction of cutting speed, as shown in Fig.13. The average distance between every two adjacent marks was defined as the length of the tool vibration mark. The values based on measurement results and the predicted values calculating by the model are shown in Fig.14 for the length of tool mark. It can be seen that there is a good agreement between the predictions based on the model and the measured values. In most cases, the prediction error was found to be within 3.5\%. However, the prediction error approached $9 \%$ when the cutting speed exceeded $500 \mathrm{~mm} / \mathrm{min}$, as shown in Fig.14 (b). It can be explained that material removal length in equations (6), (11) and (14) was longer when adopting higher cutting speed, which resulted in the reducing number of tool marks within measuring area as shown in Fig.13 (c). In this instance, the average value was not quite accurate as the tool marks were numerous as shown in Fig.13 (a). Moreover, it is found that the length of the tool vibration mark was significantly influenced by the change in cutting speed, but not by the depth of cut and vibration amplitude from Fig.14. Additionally, the higher cutting speed led to deeper marks and larger length of tool marks, as well as worse surface roughness as shown Fig.13. But the depth and length of the tool marks were too small to raise surface roughness when the cutting speed was not higher than $200 \mathrm{~mm} / \mathrm{min}$. These results are consistent with the surface roughness shown in Fig.7. It is noted that the speed of $200 \mathrm{~mm} / \mathrm{min}$ used in the $1 \mathrm{D}$ UVC system is higher than that adopted in other $2 \mathrm{D} / 3 \mathrm{D}$ vibration systems, e.g. $3 \mathrm{~mm} / \mathrm{min}$ [23]. Hence, a model for calculating the material removal length can be used to predict the length of the tool marks imprinted on the machined surface. Furthermore, the impact of tool marks on the machined surface obtained with the UVC system can be reduced and even eliminated by choosing process parameters based on the prediction model.
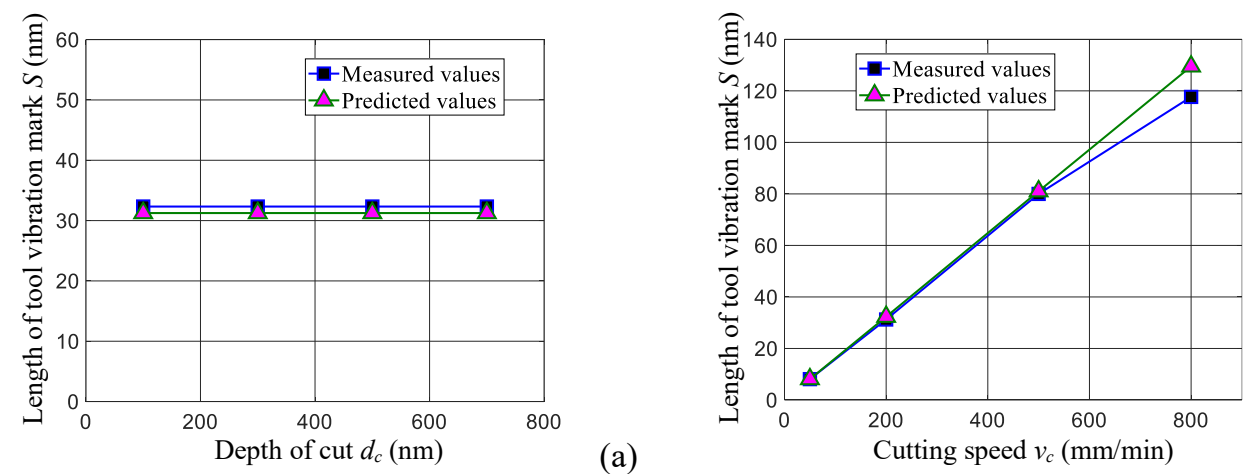


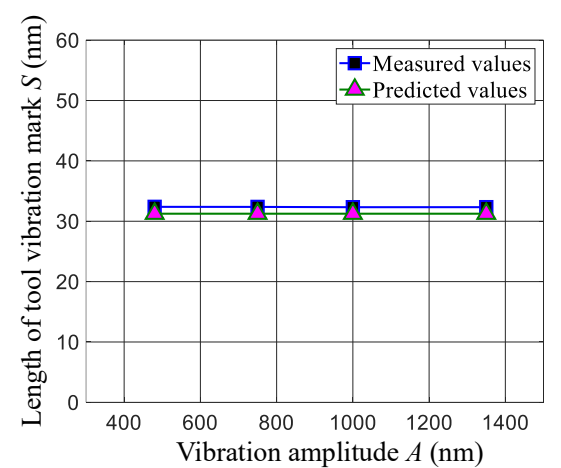

(c)

Fig.14. Relationship between the length of tool mark with process parameters (a) depth of cut, (b) cutting speed and (c) vibration amplitude.

\section{Conclusions}

Ultrasonic vibration cutting of a micro grooved silicon surface was conducted to study the critical undeformed chip thickness, characteristics of micro groove array, material removal process, and material removal mechanism. A model of the material removal length was established to predict the length of the tool vibration mark. From these studies, the following conclusions were drawn:

(1) The critical undeformed chip thickness increases with increasing vibration amplitude in the accessible range. It approaches $1030 \mathrm{~nm}$ at a vibration amplitude of $1700 \mathrm{~nm}$, which is about nine times that achieved without vibration assistance.

(2) The micro groove array on the silicon surface was generated at the plastic region with surface roughness Ra lower than $8 \mathrm{~nm}$ although the maximum value of the undeformed chip thickness reached $700 \mathrm{~nm}$. A crack free surface can be obtained only if the depth of cut is lower than the critical undeformed chip thickness and the effect of depth of cut on the surface finish is negligible. But a higher cutting speed or vibration amplitude is not beneficial for improving the surface quality.

(3) The macro chips appeared continuous, but its micro topography showed discontinuous clusters of lines with diameter in the order of dozens of nanometres. The chip displayed clusters of thin slices when the depth of cut was close to the critical undeformed chip thickness. Only polysilicon was detected in the chips due to low strain rate and compressive residual stress was found on chips obtained with higher or lower cutting speed because of unsmooth flow of the chips.

(4) A model established in this paper can be used to predict the length of the tool vibration mark. There was good agreement between the model-based predicted values and the measured values. In most cases, the prediction error was found to be within 3.5\%. Length of the tool vibration mark was significantly influenced by the change in cutting speed, but not by depth of cut and vibration amplitude.

(5) Since the depth and length of the tool marks will be too small to raise the surface roughness in ultrasonic vibration cutting when the cutting speed is not higher than $200 \mathrm{~mm} / \mathrm{min}$, the 
impact of tool marks imprinted on the machined surface can be reduced and even eliminated through choosing process parameters based on the prediction model.

\section{Acknowledgments}

The authors would like to express their sincere thanks to the National Natural Science Foundation of China (No. 51775482) and the Hebei Province Natural Science Foundation of China (No. E2016203372) for their financial support of the research work.

\section{References}

[1] D. Sreehari, A.P. Sharma. On form accuracy and surface roughness in micro-ultrasonic machining of silicon microchannels. Precision Engineering, 2018, 53: 300-309.

[2] M. Arif, M. Rahman, W.Y. San. An experimental investigation into micro ball end-milling of silicon. Journal of Manufacturing Processes, 2012, 14: 52-61.

[3] K. Katahira, K. Nakamoto, P. Fonda, et al. A novel technique for reconditioning polycrystalline diamond tool surfaces applied for silicon micromachining. CIRP Annals Manufacturing Technology, 2011, 60: 591-594.

[4] G. Romagnoli, D.A. Feito, B. Brunel, et al. Silicon micro-fluidic cooling for NA62 GTK pixel detectors. Microelectronic Engineering, 2015, 145:133-137.

[5] M. Moreno, D. Murias, J. Martı'nez, et al. A comparative study of wet and dry texturing processes of c-Si wafers for the fabrication of solar cells. Solar Energy, 2014, 101: 182-191.

[6] P. KantiBasu, A. Khanna, Z. Hameiri. The effect of front pyramid heights on the efficiency of homogeneously textured inline-diffused screen-printed monocrystalline silicon wafer solar cells. Renewable Energy, 2015, 78: 590-598.

[7] O.Y. Rogov, V.V. Artemov, M.V. Gorkunov, et al. FIB - fabricated complex - shaped 3D chiral photonic silicon nanostructures. Journal of Microscopy, 2017, 268(3): 254-258.

[8] L. Yang, A. EI-Tamer, U. Hinze, et al. Parallel direct laser writing of micro-optical and photonic structures using spatial light modulator. Optics and Lasers in Engineering, 2015, 70: 26-32.

[9] A. Pan, B. Gao, T. Chen, et al. Fabrication of concave spherical microlenses on silicon by femtosecond laser irradiation and mixed acid etching. Optics Express, 2014, 22(12): 1524515250 .

[10] Z. Yao, Y. Hu. Direct Fabrication of sub-micron sized mirco-structure by interfering nanosecond laser beams. Journal of Mechanical Engineering, 2013, 49(6): 122-127.

[11] H.K. Park, H. Onikura, O. Ohnishi, et al. Development of micro-diamond tools through electroless composite plating and investigation into micro-machining characteristics. Precision Engineering, 2010, 34: 376-386.

[12] J. Xie, Y.W. Zhuo, T.W. Tan. Experimental study on fabrication and evaluation of micro 
pyramid-structured silicon surface using a V-tip of diamond grinding wheel. Precision Engineering, 2011, 35: 173-182.

[13] J. Xie, X.R. Liu, K.K. Wu, et al. Evaluation on 3D micro-ground profile accuracy of micropyramid-structured Si surface using an adaptive-orientation WLI measurement. Precision Engineering, 2013, 37: 918- 923.

[14] J. Cheng, Y.D. Gong. Experimental study of surface generation and force modeling in micro-grinding of single crystal silicon considering crystallographic effects. International Journal of Machine Tools \& Manufacture, 2014, 77: 1-15.

[15] S.T. Chen, S.J. Lin. Study of an on-line precision microgroove generating process on silicon wafer using a developed ultra-thin diamond wheel-tool. Diamond \& Related Materials, 2011, 20: 339-342.

[16] M. Mukaida, J.W. Yan. Ductile machining of single-crystal silicon for microlens arrays by ultraprecision diamond turning using a slow tool servo. International Journal of Machine Tools and Manufacture, 2017, 115: 2-14.

[17] S.Z. Chavoshi, S. Goel, X. Luo. Influence of temperature on the anisotropic cutting behaviour of single crystal silicon: A molecular dynamics simulation investigation. Journal of Manufacturing Processes, 2016, 23:201-210.

[18] A. Mir, X. Luo, J. Sun. The investigation of influence of tool wear on ductile to brittle transition in single point diamond turning of silicon. Wear, 2016, 364-365: 233-243.

[19] Durazo-Cardenas, P. Shore, X. Luo, et al. 3D characterisation of tool wear whilst diamond turning silicon. Wear, 2007, 262: 340-349.

[20] X.Q. Zhang, M. Arif, K. Liu, et al. A model to predict the critical undeformed chip thickness in vibration-assisted machining of brittle materials. International Journal of Machine Tools and Manufacture, 2013, 69: 57-66.

[21] J.G. Zhang, T. Cui, C. Ge, et al. Review of micro/nano machining by utilizing elliptical vibration cutting. International Journal of Machine Tools and Manufacture, 2016, 106: 109126.

[22] Z.W. Zhu, S. To, G.B. Xiao, et al. Rotary spatial vibration-assisted diamond cutting of brittle materials. Precision Engineering, 2016, 44: 211-219.

[23] J. Wang, Y. Yang, P. Guo. Effects of vibration trajectory on ductile-to-brittle transition in vibration cutting of single crystal silicon using a non-resonant tool. Procedia CIRP, 2018, 71: 289-292.

[24] D.E. Brehl, T.A. Dow. Review of vibration-assisted machining. Precision Engineering, 2008, 32: 153-172.

[25] T. Moriwaki, E. Shamoto, K. Inoue, et al. Ultraprecision Ductile Cutting of Glass by Applying Ultrasonic Vibration. CIRP Annals, 1992, 41(1): 141-144.

[26] H. Saito, H. Jung, E. Shamoto, et al. Mirror surface machining of high-alloy steels by elliptical vibration cutting with single-crystalline diamond tools: Influence of alloy elements on diamond tool damage. Precision Engineering, 2017, 49: 200-210. 
[27] C. Ma, Z. Wang. Experimental and numerical investigation of the breakage of a cutting tool with ultrasonic vibration. Precision Engineering, 2018, 51:393-402.

[28] W. Zong, T. Sun, D. Li, et al. Tool wear mechanism involved in diamond turning of single crystal silicon. Nanotechnology and Precision Engineering, 2009, 7(3): 270-274.

[29] F. Xu, X. Zhang, F. Fang. Characterization of single point diamond machined single-crystal silicon. Nanotechnology and Precision Engineering, 2013, 11(6): 485-491.

[30] M.J. Klopfstein, R. Ghisleni, D.A. Lucca, et al. Surface characteristics of microultrasonically machined $\left(\begin{array}{lll}1 & 0 & 0\end{array}\right)$ silicon. International Journal of Machine Tools and Manufacture, 2008, 48: 473-476. 